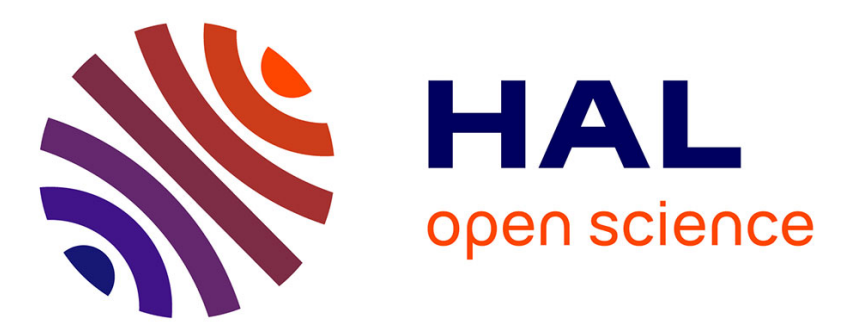

\title{
A contrast equalization procedure for change detection algorithms: applications to remotely sensed images of urban areas
}

\author{
Alexandre Fournier, Pierre Weiss, Laure Blanc-Féraud, Gilles Aubert
}

\section{- To cite this version:}

Alexandre Fournier, Pierre Weiss, Laure Blanc-Féraud, Gilles Aubert. A contrast equalization procedure for change detection algorithms: applications to remotely sensed images of urban areas. International Conference on Pattern Recognition, International Association of Pattern Recognition, Dec 2008, Tampa, United States. pp.1-4. inria-00307727

\section{HAL Id: inria-00307727 https://hal.inria.fr/inria-00307727}

Submitted on 29 Jul 2008

HAL is a multi-disciplinary open access archive for the deposit and dissemination of scientific research documents, whether they are published or not. The documents may come from teaching and research institutions in France or abroad, or from public or private research centers.
L'archive ouverte pluridisciplinaire HAL, est destinée au dépôt et à la diffusion de documents scientifiques de niveau recherche, publiés ou non, émanant des établissements d'enseignement et de recherche français ou étrangers, des laboratoires publics ou privés. 


\title{
A contrast equalization procedure for change detection algorithms: applications to remotely sensed images of urban areas
}

\author{
Alexandre Fournier ${ }^{\dagger}$, Pierre Weiss ${ }^{\dagger}$, Laure Blanc-Féraud ${ }^{\ddagger}$ \\ Ariana Research Group - INRIA/I3S, CNRS \\ $\left\{{ }^{\dagger}\right.$ FirstName.LastName, ${ }^{\ddagger}$ laure.blanc_feraud\}@ @ophia.inria.fr \\ Gilles Aubert, Laboratoire Dieudonne - UMR 6621 CNRS \\ gaubert@math.unice.fr
}

\begin{abstract}
We propose an algorithm that equalizes the contrast of grayscale image pairs to simplify the task of change detection. To ensure robustness of the detection under different illumination conditions, some authors recently proposed algorithms that compare the level lines of the images. We show - using ideas from the "shape from shading" community - that under directed light, a necessary condition for the level lines to be illumination invariant is that the underlying surfaces be developable. The surfaces of cities can be modeled as piecewise smooth developable surfaces, and it is therefore sensible to make use of the level lines for change detection. Our algorithm is robust and efficient both on synthetic OpenGL scenes and natural Quickbird images.
\end{abstract}

\section{Introduction}

Supervised and unsupervised change detection algorithms are now crucial in satellite imagery. Huge volumes of data are regularly collected and human inspection can be greatly facilitated with automated processing. One of the principal difficulties to design an algorithm that only detects pertinent changes is that the scenes are generally taken under different illumination conditions. Therefore the detection should not be based on a comparison of pixel intensity but rather on illumination-invariant features. Caselles et al. proposed such invariant features in [3]: the level lines. These can be shown to be invariant when the intensity of the light varies but not when the direction of incidence of the light varies. Figure (1) clearly illustrates this fact. In remotely sensed applications, the light is directed (it comes from the sun) and it therefore seems unappropriate to use level lines. We show that level lines are actu- ally illumination-invariant provided that the 3-D scene surface satisfies some geometrical properties. Then we show that those properties are almost met in urban areas. This leads us to propose a simple algorithm that "equalizes" the contrast of both images using a tool close to the level lines: the connected components of the isolevels. After this pre-processing, a simple difference gives promising results both on synthetic and natural images.


Figure 1. Top: images of a 3-D dome illuminated with Phong model [5] using two different incident light directions. Bottom: some of their level lines.

\section{Notations and hypotheses}

\subsection{Notations}

Let us introduce some notations illustrated in Figure 2. $\Omega$ represents the image plane. $S: \mathbb{R}^{2} \rightarrow \mathbb{R}$ designates the scene elevation. $N(X, Y)$ represents the normal to the scene surface at point $(X, Y, S(X, Y))$. $P:(X, Y, Z) \mapsto(x, y)$ is a perspective projection on $\Omega . p$ is the application defined by: 


$$
\begin{aligned}
& p: \mathbb{R}^{2} \rightarrow \mathbb{R}^{2} \\
& (X, Y) \mapsto P(X, Y, S(X, Y))
\end{aligned}
$$

We suppose that $p$ is bijective (the camera can see all points of the surface) and that $p^{-1}$ is $C^{1} . l$ is a vector in $\mathbb{R}^{3} \backslash\{0\} \cdot \frac{l}{|l|}$ denotes the direction of incidence of the light and $|l|$ denotes its intensity.



Figure 2. Notations

\subsection{Hypotheses on the surface and the light}

To model the interactions between a surface and the light, we use the Phong reflectance model [5]. We make the following hypotheses on the light and the surface:

Hypotheses: We consider that the light is composed of ambient light (light present everywhere uniformly in the scene) and directed light (all light rays are parallel with equal intensity). To avoid the presence of shadows we suppose that the angle between the $l$ and $N$ is stricly less than $\pi / 2$. Furthermore we suppose that the surface is Lambertian with constant albedo.

With these hypotheses the image $u$ of the scene $S$ under light $l$ can be written as:

$$
u(S, l)(x, y)=\left\langle l, N\left(p^{-1}(x, y)\right)\right\rangle+c s t
$$

The first term models the effect of the directed light. cst is a constant that depends on the albedo of the surface. It models the effect of the ambient light.

\section{Level line invariance}

Some recent algorithms make large use of the level lines to ensure illumination invariance $[1,4]$. They yield good results, but examples as Figure (1) show that they are not fully justified. In this part we show that if the surface respects some geometrical properties then the level lines are illumination-invariant features.
Let us first recall the definition of level lines. In [3], the authors define the level sets of an image $u: \mathbb{R}^{2} \rightarrow \mathbb{R}$ as the sets $\left\{(x, y) \in \mathbb{R}^{2}, u(x, y) \leq \lambda\right\}$. The boundaries of their connected components are the level lines of $u$. We use a slightly different definition:

Definition: The level lines are defined as the connected components of the isolevels $\{(x, y) \in$ $\left.\mathbb{R}^{2}, u(x, y)=\lambda\right\}$.

If $u$ is $C^{1}$ and if its gradient does not vanish, both definitions are equivalent. Let us analyze the level line invariance when the surface is smooth and non-smooth.

\subsection{Smooth case}

In this section, we suppose that $S$ is $C^{2}$. This implies that $u(S, l)$ is $C^{1}$ for any $l$. Let $u_{1}$ and $u_{2}$ be two $C^{1}$ images with not vanishing gradient. They have the same level lines iff $\nabla u_{1}(x, y)$ and $\nabla u_{2}(x, y)$ are colinear $\forall(x, y) \in \mathbb{R}^{2}$. This remark motivates the following result (proof in a forthcoming research report):

Proposition 1: A necessary and sufficient condition for $\nabla u\left(S, l_{1}\right)(x, y) / / \nabla u\left(S, l_{2}\right)(x, y), \forall\left(l_{1}, l_{2}\right)$ is that the Hessian of $S$ at point $(x, y)$ possesses a null eigenvalue.

This proposition indicates that for the level lines to be invariant with respect to the light direction of incidence, the surface must have a zero Gaussian curvature on every point. Such surfaces are called developable [6]. Simple examples of such objects are planes, cylinders and cones. A developable surface has the following properties [6]: each point of the surface lies on a line (the generatrix) that belongs to the surface. Furthermore the tangent plane is the same on each point of the generatrix. Thus the intensity of the reflected light is the same on every point belonging to the same generatrix. This remark implies that the projections of the generatrices on the image plane constitute isolevels of $u(S, l)$ for any $l$.

\subsection{Non smooth case}

In the former section, we have shown that, provided the surface $S$ is $C^{2}$, developable, with constant albedo, its level lines are invariant to an illumination change. Those assumptions are far too restrictive if we aim at finding changes in images of urban scenes. The albedo clearly varies. Furthermore $S$ can only be considered $C^{0}$ as discontinuities of its derivative appear in all interfaces between walls and roofs for instance.

This leads us to analyze the invariance of the level lines when $S$ is a piecewise developable $C^{2}$ mapping and that the albedo is constant on each piece. This new model fits quite well to most of the urban scenes. 
We define $L_{l}(x)$ as the level line of $u(S, l)$ passing through point $x(x \in \Omega)$. Given these assumptions, we can assert the following proposition:

Proposition 2: Let $\omega_{i}$ and $\omega_{j}$ be adjacent pieces. Two adjacent level-lines of $\left.u(S, l)\right|_{\omega_{i}}$ and $\left.u(S, l)\right|_{\omega_{j}}$ merge for almost no $l$.

Figure (3) illustrates why the equality is true only for "almost every" light orientation. The triangle-shaped roof is composed of two plane portions. If the light direction belongs to the plane bisecting these portions, then they will have the same radiosity. For most light orientations, the roof will thus be constituted of two level lines (yellow and red), while for a set of zero measure, it can be constituted of only one level set (red). Most of the level lines of the cylinder-shaped roof are just segments on the roof, but depending on the light orientation, one or two of these segments can merge with the "building wall" and create non invariant level lines.
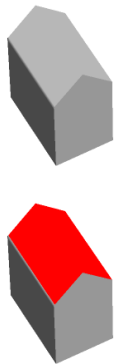
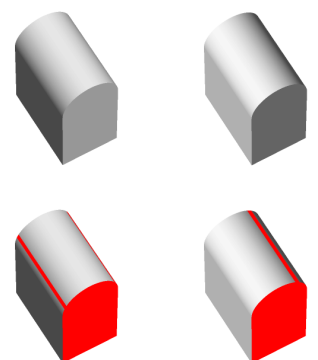

Figure 3. Examples of non invariance of the level lines in the non-smooth case. The colored parts represent singular level lines.

\section{An algorithm for contrast equalization}

We saw that the level lines of urban area images should be "almost" invariant to illumination changes. We propose a contrast enhancement and a change detection procedure that take advantage of this result. Let $u_{1}$ and $u_{2}$ be two exactly registered images taken under different lighting conditions $l_{1}$ and $l_{2}$ at times $t_{1}$ and $t_{2}$. Let $S_{1}$ be the 3-D scene at time $t_{1}$. Under these assumptions we can write:

$$
\left\{\begin{array}{l}
u_{1}=u\left(S_{1}, l_{1}\right) \\
u_{2}=u\left(S_{1}, l_{2}\right)+c_{1,2}
\end{array}\right.
$$

where $c_{1,2}$ denotes the changes from image $u_{1}$ to image $u_{2}$. In this equation $u\left(S_{1}, l_{2}\right)$ and $c_{1,2}$ are unknown. To retrieve them, we can introduce priors. From the previous discussion, it is natural to consider that $u\left(S_{1}, l_{2}\right)$ should belong to the space of images which have the same level lines as $u_{1}$. We denote this space $\chi_{u_{1}}$. We can also devise a prior on the changes $J(c)$. In most applications, the changes are sparse. In this paper, as the $L^{1}$-norm is well known to favor sparse structures, we simply set $J(c)=\|c\|_{1}$. To retrieve $c_{1,2}$ we can thus solve the following problem:

$$
\inf _{u \in \chi_{u_{1}}}\left(\left\|u_{2}-u\right\|_{1}\right)
$$

and set $c_{1,2}=u_{2}-\bar{u}$ where $\bar{u}$ is the solution of (4). Problem (4) can be reformulated as follows : "find the image $u$ closest to $u_{2}$ which has the same level lines as $u_{1}$ ". It is therefore a problem of contrast equalization. To solve (4) we need to discretize $\chi_{u_{1}}$. We propose the following simple strategy:

1. Set $u_{Q}=\left\lfloor\frac{u_{1}}{\Delta}\right\rfloor \Delta$ (uniform quantization).

2. For each level $k \Delta(k \in \mathbb{Z})$, separate the connected components $\Omega_{k, j}$ of the set $\Omega_{k}=\{x \in$ $\left.\mathbb{R}^{n}, u_{Q}(x)=k \Delta\right\}$. In the experiments, we use the 8-neighbourhood to define the notion of connected component.

We define $\chi_{u_{1}}$ as the set of images that are constant on each set $\Omega_{k, j}$. With this definition, the solution of (4) is in closed form:

$$
\left.\bar{u}\right|_{\Omega_{k, j}}=\operatorname{median}\left(\left.u_{2}\right|_{\Omega_{k, j}}\right)
$$

This kind of algorithm has already been used and analyzed with a different motivation in [2]. This is a very fast algorithm (less than 0.4 second for a $1000 \times 1000$ image on an Intel Xeon CPU @ 1.86GHz).

\section{Results}

\subsection{Synthetic images}

To outline the results presented in this paper, we devised a simple 3-D scene generator, which allows one to visualize simple instances of cities under different ligthing conditions. The top images in Figure (4) show two images of urban areas. In this example, some buildings appeared or disappeared, the shape of some elements changed and some buildings moved. Clearly, no algorithm based on a global contrast change (histogram equalization for instance) can provide satisfying results on such images. The output of our algorithm is almost perfect except on the dome (Gaussian curvature is not null), and for a few level lines. 

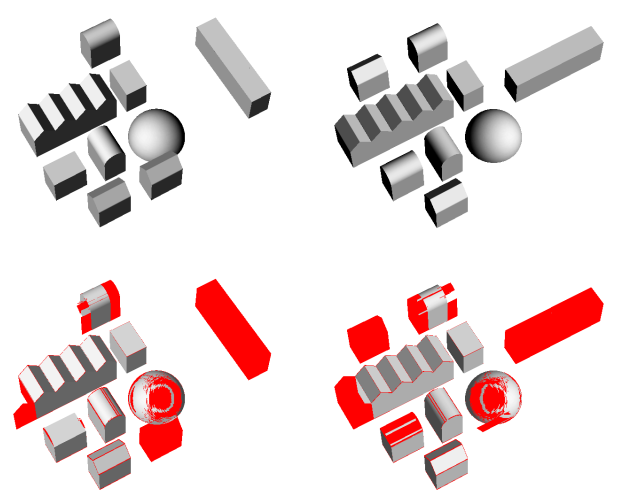

Figure 4. Toy example. Top: two images under different lighting conditions with some changes. Bottom: the red parts correspond to the structures detected as changed by our algorithm.

\subsection{Natural images}

Let us now turn to real images. Our assumptions on the scene surface are only met at large scales. The roof tiles, for instance, can seldom be considered as developable, whereas the whole roof can. To apply the previous algorithm, we thus begin by a fast cartoon+texture decomposition algorithm [8] and only work on the cartoon parts. Furthermore we have not considered shadows in our model. Shadowed regions are only lightened by ambient light. Their intensity can generally be considered as 10 times lower than the regions lightened by directed light [7]. In Figure (5) we thus remove the changes due to shadows by not considering the low intensity changes. Compared with a classical approach (i.e. global contrast equalisation followed by a per-pixel difference, bottom left), this simple algorithm yields satisfying results (bottom right). In this example both methods yield a $75 \%$ true positive rate. The false positive rate is of $25 \%$ for our method and $60 \%$ for the global contrast change. The main reason for failure is the problem of parallax: the images are registered rigidly, but they are not taken from the same location.

\section{Conclusion}

We analyzed the behavior of the level lines of an image when the light direction varies. We have shown that they are "almost" invariant if the scene surface is piecewise developable. Based on this result, we proposed a simple and fast contrast equalization algorithm. With this algorithm, simple differences give promising results on real images. Further work will include more complex change detection rules. For lack of space, we
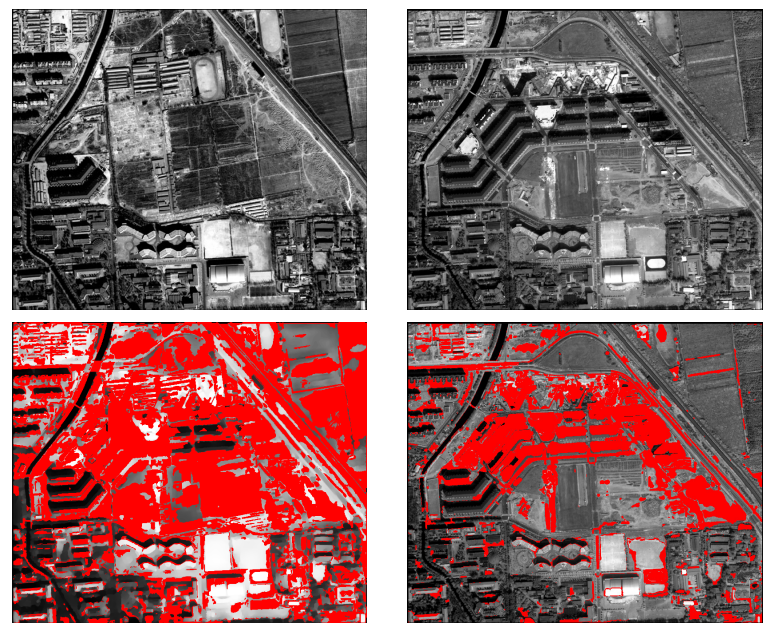

Figure 5. Top: Quickbird images (resolution $61 \mathrm{~cm}$ ) of Beijing in 2001 and 2003. BL: change detection result after global contrast equalization. BR: change detection result using the proposed approach.

will provide the proofs of the propositions, comparisons with other algorithms and more detailed comments in a forthcoming research report.

Acknowledgments: The authors would like to thank Véronique Prinet for fruitful discussions and the RSIU team at Liama laboratory, Beijing, for kindly providing the high resolution image pairs. The first author would like to thank the French Defense Agency (DGA) for partial funding of his $\mathrm{PhD}$.

\section{References}

[1] C. Ballester, E. Cubero-Castan, M. Gonzalez, and J. Morel. Contrast invariant image intersection. Advanced Mathematical Methods in Measurement and Instrumentation, pages 41-55, 2000.

[2] V. Caselles, B. Coll, and J. Morel. Geometry and Color in Natural Images. JMIV , 16:89-107, 2002.

[3] V. Caselles, B. Coll, and J.-M. Morel. Topographic Maps and Local Contrast Changes in Natural Images. International Journal of Computer Vision, 33, 1999.

[4] P. Monasse and F. Guichard. Fast computation of a contrast-invariant image representation. IEEE Trans. on Image Proc., 9:860-872, 2000.

[5] B. T. Phong. Illumination for computer generated pictures. Comm. ACM, 18(6):311-317, June 1975.

[6] M. Spivak. A comprehensive Introduction to Differential Geometry, volume 3. Publish or Perish, Inc., 1999.

[7] S. Watanabe, K. Miyajima, and N. Mukawa. Detecting changes of buildings from aerial images using shadow and shading model. In ICPR, pages 1408-1412, 1998.

[8] P. Weiss, L. Blanc-Féraud, and G. Aubert. Efficient schemes for total variation minimization under constraints in image processing. RR 6260, INRIA, July 2007. 